$I^{2} M T C 2008$ - IEEE International Instrumentation and Measurement Technology Conference

Victoria, Vancouver Island, Canada, May 12-15, 2008

\title{
A Novel 24 GHz One-Shot, Rapid and Portable Microwave Imaging System
}

\author{
M.T. Ghasr ${ }^{1}$, M.A. Abou-Khousa ${ }^{1}$, S. Kharkovsky ${ }^{1}$, R. Zoughi ${ }^{1}$, and D. Pommerenke ${ }^{2}$ \\ 'Applied Microwave Nondestructive Testing Laboratory (amntl) \\ 'Electromagnetic Compatibility Laboratory \\ Electrical and Computer Engineering Department \\ Missouri University of Science and Technology (Missouri S\&T) \\ (formerly University of Missouri-Rolla), Rolla, Missouri 65409, USA \\ Phone: (573) 341-4728, Fax: (573) 341-6671 \\ Email: (mtg7w6; maamc2; sergiy; zoughi; pommerenke)@mst.edu
}

\begin{abstract}
Development of microwave and millimeter wave imaging systems has received significant attention in the past decade. Signals at these frequencies penetrate inside of dielectric materials and have relatively small wavelengths. Thus, imaging systems at these frequencies can produce images of the dielectric and geometrical distributions of objects. Although there are many different approaches for imaging at these frequencies, they each have their respective advantageous and limiting features (hardware. reconstruction algorithms). One method involves electronically scanning a given spatial domain while recording the coherent scattered field distribution from an object. Consequently, different reconstruction or imaging techniques may be used to produce an image (dielectric distribution and geometrical features) of the object. The ability to perform this accurately and fast can lead to the development of a rapid imaging system that can be used in the same manner as a video camera. This paper describes the design of such a system, operating at $24 \mathrm{GHz}$, using modulated scatterer technique applied to 30 resonant slots in a prescribed measurement domain.
\end{abstract}

Keywords - electric field distribution, modulated scatterer technique, resonant slots, $2 D$ array, microwave imaging

\section{INRODUCTION}

Microwave imaging is based on measuring the relative scattered field from an object over a known two-dimensional (2D) space and then using various imaging algorithms (i.e., synthetic aperture, holographical, reconstruction techniques, etc.) to obtain geometrical and dielectric distribution of the object (or essentially its image) [1-2]. To this end, an individual scanning and non-perturbing probe or an array of probes capable of measuring the magnitude and phase of the scattered electric field component of interest is used to obtain the coherent electric field distribution over the 2D space (i.e., mapping the field) at certain prescribed spatial intervals. The basic probe design requires that its deployment must not significantly perturb the scattered field to be measured. Furthermore, the probe should be robust against parasitic reflections and coupling between probe array elements [2-3]. A probe, which meets the above design requirements, is one employing the modulated scatterer technique (MST). The use of MST for electric field measurements was first introduced in [3]. Since its introduction, MST has been incorporated into various linear scatterers, such as small dipole antennas, and has been extensively used for electric field mapping and imaging applications.

MST-based 2D imaging systems have been designed and built with an array of sub-resonant dipoles at frequencies up to $12 \mathrm{GHz}$ (X-band) [2,4]. Although such designs use minimally perturbing elements and result in a compact array for field sampling and measurement, they suffer from several drawbacks. The inefficiency of the sub-resonant dipoles, places their scattered signal very close to the noise floor of most detection systems. Furthermore, the mutual coupling among the dipoles can significantly limit system dynamic range. These problems become even more significant and challenging at higher frequencies such as those in the millimeter wave region $(30-300 \mathrm{GHz})$ where currently there is a great need for rapid imaging systems at these frequencies.

In this paper, a novel MST-based 2D imaging system capable of rapid electric field mapping, operating at $24 \mathrm{GHz}$ (K-band), is discussed. The design is versatile enough to be directly implemented at higher frequencies. Modulated scatterer technique allows for spatial tagging of the desired electric field facilitating the evaluation of magnitude and phase of a scattered field at distinct and localized locations within a 2D spatial domain (i.e., the probe array). In other words, when the receiver demodulates the MST signal, it gives an output proportional to the magnitude and phase of the incident (or scattered) electric field at the location of the modulated probe. Therefore, an array of modulated probes provides for coherent electric field distribution over the desired 2D space. Another important feature of MST is that the effect of other non-coherent signals such as clutter and noise, adversely influencing the measurement, is significantly reduced through various means including coherent averaging [2]. The main feature of the proposed array design is high-Q compact resonant slots that are loaded with PIN diodes, and are then modulated either individually (i.e., sequentially) or simultaneously using orthogonal codes. To illustrate the efficacy of this design, a prototype array of 30 slots ( 6 by 5 ) with an element spacing of $\lambda_{0} / 2$, where $\lambda_{0}$ is the free-space wavelength, and operating at a frequency of $24 \mathrm{GHz}$ was designed and constructed. A schematic of this prototype is shown in Figure 1. 


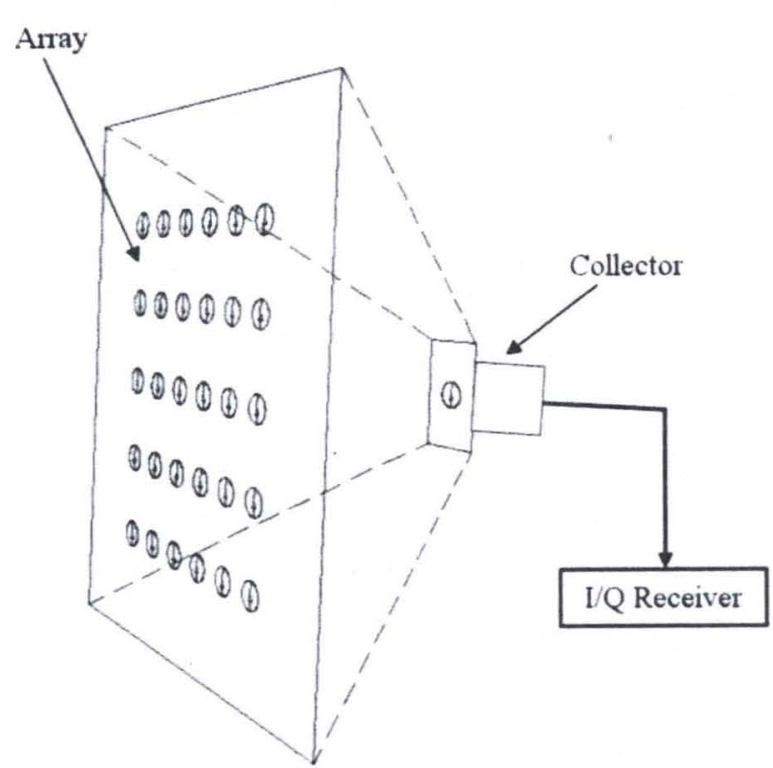

Fig. 1. Schematic of the microwave imaging system with the PIN diodeloaded slot array.

When designing a rapid MST-based imaging system the two critical parameters that must be carefully considered and designed for are the switching speed and the modulation depth. Modulation depth refers to the difference between the detected signal magnitudes when the PIN diode is on and when it is off. The larger this value is, the higher the system sensitivity and the overall signal-to-noise ratio $(\mathrm{S} / \mathrm{N})$ will be. To satisfy these requirements, high-Q resonant slots loaded with a fast switching PIN diode were used in the array design. The PIN diode used in this design is capable of a switching time in the order of $3 \mathrm{nS}$, during which the slot changes from having a very low insertion loss $(-20 \mathrm{~dB})$ value to having a very high insertion loss $(-2 \mathrm{~dB})$ value, corresponding to when the diode is off and on (slot being open and short circuited), providing a relatively large modulation depth with a relatively fast switching speed satisfying the desired requirements for a robust design associated with this type of electric field mapping. As mentioned earlier, the large modulation depth and the fact that coherent averaging over several modulation periods is readily possible, provides the system with a relatively high $\mathrm{S} / \mathrm{N}$. In addition, the salient features of the slots are that they are efficient and exhibits very small mutual coupling [5]. Mutual coupling is of course also dependent on the relative placing of the slots next to one another. Although, the largest dimension of the slot is less than $\lambda_{0} / 3$, it is made to be resonant at the frequency of interest by special loading. Slot spacing of $\lambda_{\mathrm{o}} / 2$ ensures adequate sampling of the field without sacrificing signal fidelity, which is adversely affected by the mutual coupling $|5|$. The probe array, as shown in Figure 1, was manufactured using standard photolithographic PCB manufacturing technique on a Rogers 4350 board of 0.020 " $(0.5 \mathrm{~mm})$ thickness. Figure 2 shows a picture of the array with the 30 slots showing.

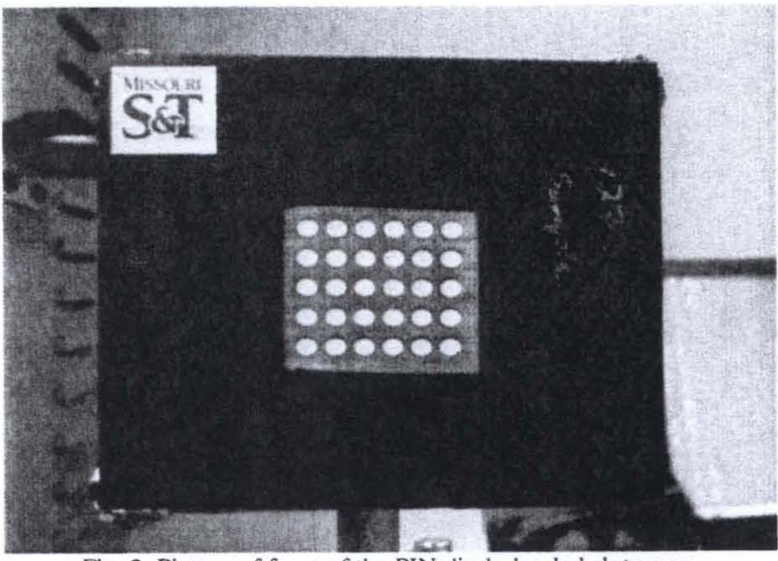

Fig. 2. Picture of front of the PIN diode-loaded slot array.

The electric field scattered by the object to be imaged reaches the plane of the array and the respective electric fields at the particular location of each slot is then coupled into that slot. Subsequently, this signal (picked up by the slot) is re-radiated to the opposite side of the array. Using the PIN diodes, each slot is modulated at a certain modulation rate. In this way the electric field due to each slot can be individually discriminated and measured. This is the basis for using MST so that the scattered field from an object over a planar spatial domain can be unambiguously determined. To pickup the modulated scattered field from each slot, a collector consisting of a $\mathrm{K}$-band rectangular waveguide with a resonant slot at its aperture is placed behind the array screen. This collector has the advantages of having a very small insertion loss, and a wide radiation pattern engulfing the array within its main lobe enabling signals from each slot to be detected and picked up. Having a single collector allows the use of a single receiving RF front-end without the use of complex microwave combiners. The combination of a relatively large planar array and a small collector, as shown in Figure 1, results in a non-uniform coupling coefficients between each array element (slot) and the collector both in magnitude and phase. To correct for this, a complex coefficients for each slot array element is measured by irradiating the array with a uniform plane-ivave. Since the measured field has a known distribution (i.e., a uniform magnitude and phase distribution), any variation (or distortion) from that norm, is then caused by the array. Consequently, any subsequent measurement is referenced to the uniform plane wave measurement (i.e., calibration). Finally, an I/Q detector is used to measure the magnitude and phase of the captured reradiated signal by each slot.

\section{MEASUREMENT DISCUSSIONS}

System dynamic range is a critical parameter influencing and illustrating the sensitivity to detecting small electric field values and combined with large modulation depth it enables distinguishing among small differences in the measured values in adjacent slots. Figure 3 shows the measured dynamic range of the receiver front-end. The results show a 
70- $\mathrm{dB}$ dynamic range as well as the desired receiver linearity required for sensitive imaging systems.

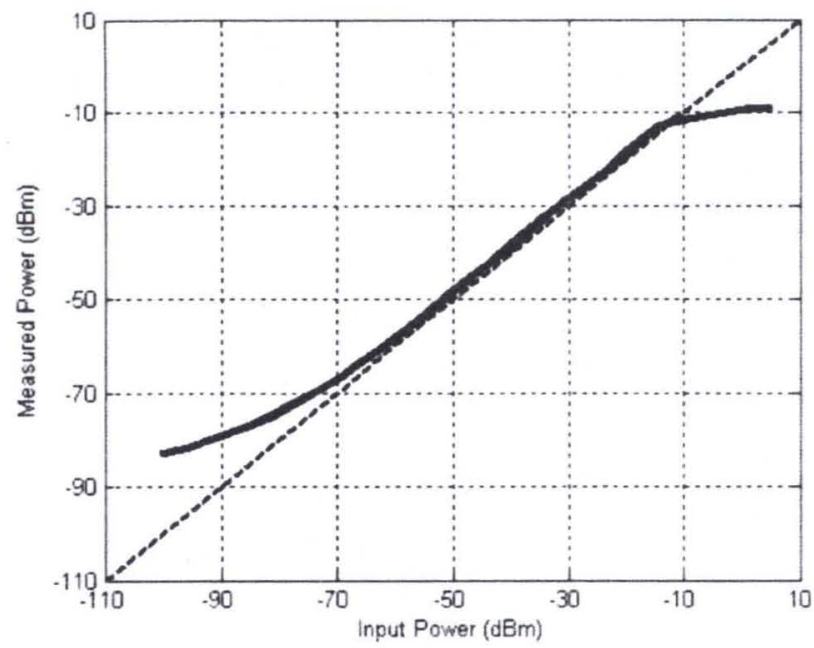

Fig. 3. Dynamic range of the receiver's RF front end showing the saturation level, noise floor, and deviation from an ideal case.

Several experiments were performed to assess the performance of the array probe. One of these experiments was set up as a through-transmission configuration where the probe array was used as a receiver in the far-field region of a transmitting open-ended $\mathrm{K}$-band rectangular waveguide. The distance between the transmitter and the array was $80 \mathrm{~mm}$. The electric field distribution due to the incident field radiated by the transmitting open-ended waveguide was measured and used as a reference to correct for the distortion caused by the array probe, as explained earlier. Then, a small metallic sphere $(4 \mathrm{~mm}$ in diameter) was placed between the rectangular waveguide transmitter and the array probe receiver. Figure 4 shows the measurement set up.

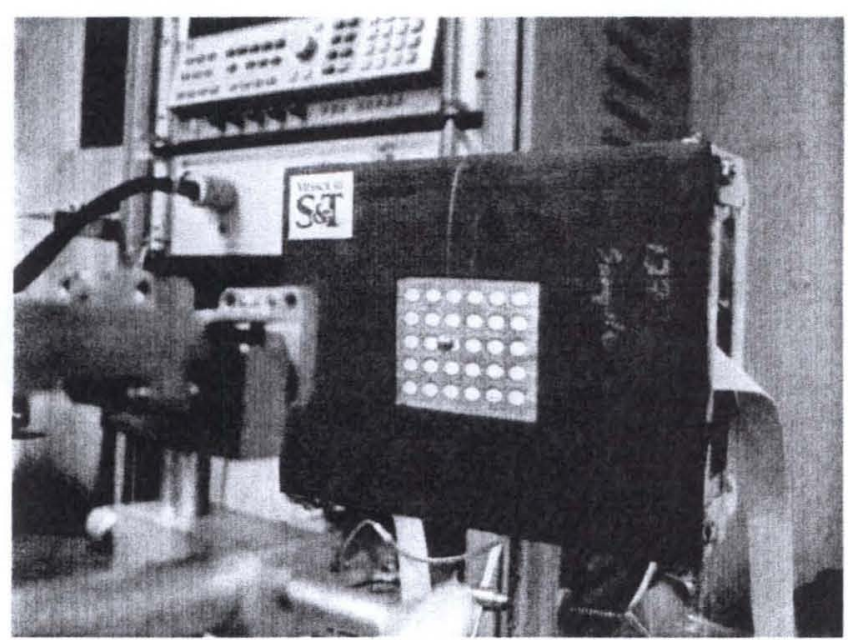

Fig. 4. Picture of the measurement set up showing the waveguide transmitter, a small metallic sphere in front of the array, and the slot array.

Figure 5 shows the measured electric field distributions when the sphere is located $5 \mathrm{~cm}$ and $12 \mathrm{~cm}$ away from the slot array. Due to the limited number of sampling points (total of 30 pixels) of the probe array, the field distributions shown in Figure 5 was up-sampled 20 times and spatially interpolated to produce a smoother and a more representative image of the electric field distribution. It must be noted that these images were measured and processed in real-time (under 15 milliseconds), which demonstrate the capability of this prototype imaging system to produce images rapidly, which is a major practical requirement for most applications. The indication of the sphere can be clearly seen in Figure 5a as a low intensity region showing the sphere casting its shadow on the array. This shadow indication is much larger than the sphere itself due to the distance from the sphere to the array. Furthermore, this indication is not circularly symmetric due to the rectangular geometry and limited number of sampling points on the array. Taking the sphere further away from the imaging array causes the sphere indication to appear larger, as expected (Figure $5 \mathrm{c}-\mathrm{d}$ ). These are indications of raw data. Of course using imaging algorithms one can get a much better and a more closely representative indication of the sphere. In addition, there is a high intensity spot in the center of the image shown in Figure $5 \mathrm{c}$ which is probably an indication of creeping waves since the small size of the sphere $(2 \pi \mathrm{r} / \lambda=1)$ places it in the Mie scattering region.

In another experiment, the system is operated in the reflection mode in which the probe array was used as the transmitter and the receiver. A metallic sphere, $10 \mathrm{~mm}$ in diameter was placed at a distance of $10 \mathrm{~mm}$ in front of the array. Figure 6a-b shows the magnitude and phase of the scattered electric field off the sphere. Due to the limited resolution of the array, the images were up-sampled 6.25 times to produce an image of one pixel per $\mathrm{mm}^{2}$. Subsequently, this data was used to produce a synthetic aperture focused (SAF) image of the sphere, as shown is Figure $6 \mathrm{c}[1]$. The results clearly show a more representative image (a $10 \mathrm{~mm}$ diameter indication) of the sphere. Various image processing algorithms, such as synthetic aperture method, can be applied to the collected data as per the requirement of the applications $[4,6-9]$.

\section{SUMMARY}

A novel 2D microwave imaging system at $24 \mathrm{GHz}$ based on MST techniques utilizing high-Q resonant slots loaded with PIN diodes and a 30-element array probe prototype was designed and constructed. This imaging system is capable of producing images at a rate in excess of 30 images per second. The results have demonstrated the utility of the imaging system for electric field distribution mapping and high fidelity real-time imaging. Using this array probe not only the electric field distribution of scatterers, but also the near and/or far electric field distribution of various radiating elements and structures can be mapped rapidly. This paper presented the basic design of the imaging system along with a few measurement results. 


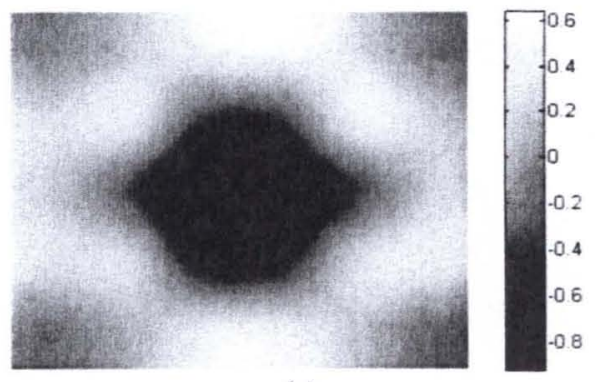

(a)

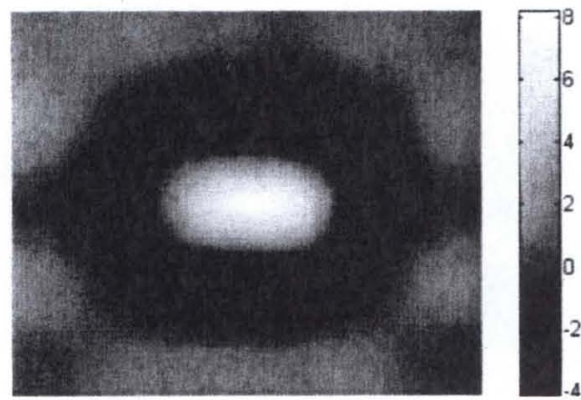

(b)

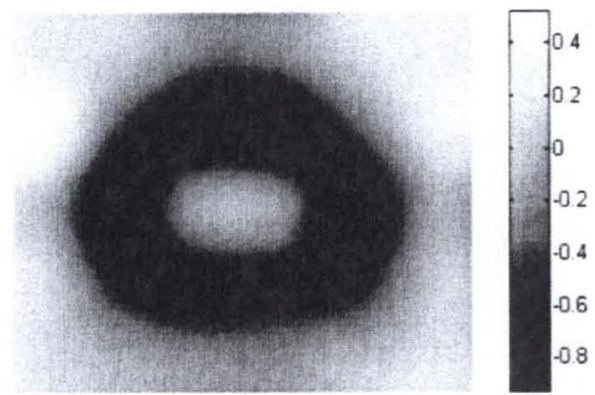

(c)

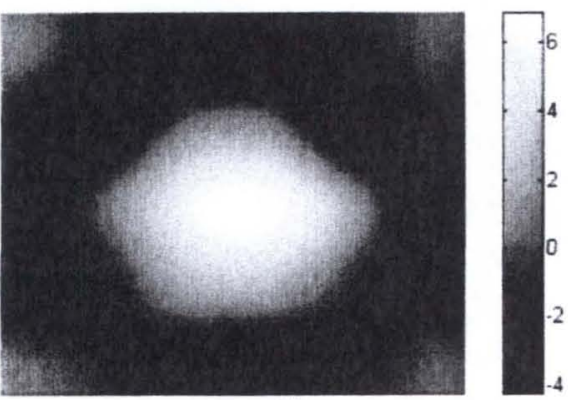

(d)

Fig. 5. Magnitude (dB) and phase (deg.) of measured scattered electric field from a 4-mm diameter metallic sphere at a distance of $5 \mathrm{~mm}$ from the array: a) magnitude, b) phase, and at a distance $12 \mathrm{~mm}$ from the array: c) magnitude, d) phase.

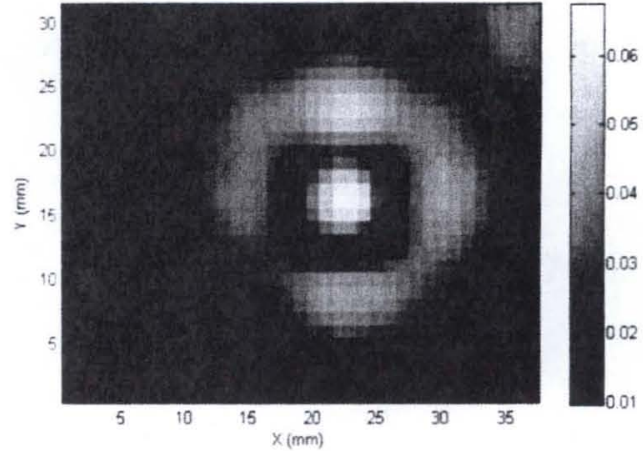

(a)

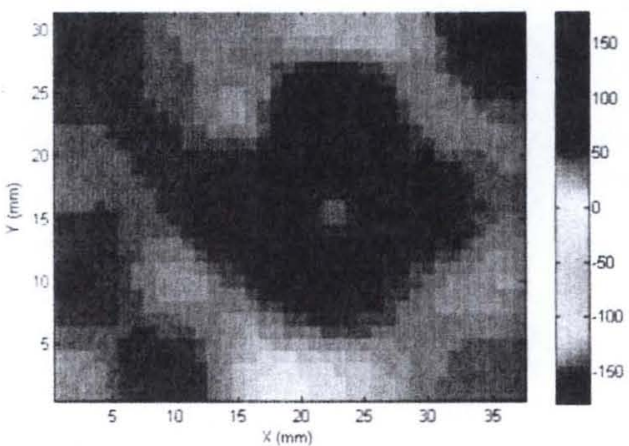

(b)

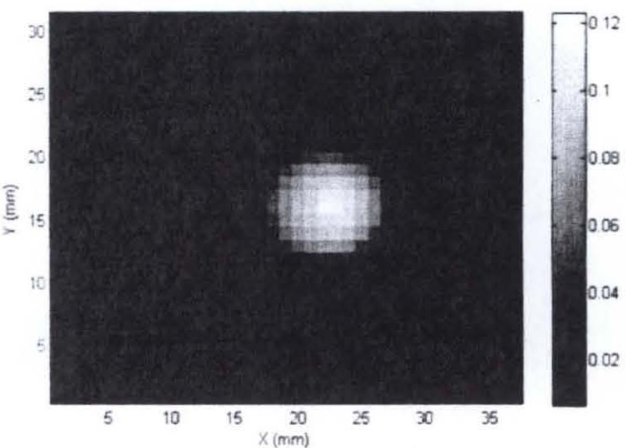

(c)

Fig. 6. (a) Magnitude and (b) phase (deg.) of measured scattered electric field from a metallic sphere $10-\mathrm{mm}$ in diameter at a distance of $10 \mathrm{~mm}$ from the array in reflection mode, (c) the data is SAF processed to produce precise image of the sphere.

\section{ACKNOWLEDGEMENT}

This works was supported by a grant from the NASA Marshall Space Flight Center (MSFC), Huntsville, AL, USA.

\section{REFERENCES}

11 J.T. Case, F. Hepburn and R. Zoughi, "Inspection of spray on foam insulation (SOFI) using microwave and millimeter wave synthetic aperture focusing and holography," in Proc. IEEE Instrum. Meas. Teclinol. Conf. , Sorrento, Italy, , 2006. pp. 2148-2153 
[2] J.-C. Bolomey, G.E. Gardiol, "Engineering applications of the modulated scatterer technique," Norwood, MA: Artech house Inc. 2001.

[3] J. H Richmond, "A modulated scattering technique for measurement of field distributions," IEEE Trans.Microw. Theory Tech.., vol. 3, no. 4, pp. 13-15, Jul. 1955.

[4] A. Franchios, A. Joisel, C. Pichot, and J.-C. Bolomey, "Quantitative microwave imaging with a $2.45-\mathrm{GHz}$ planar microwave camera," IEEE Trans. Medical Imaging, vol. 17, no. 4, pp. 550-561, Aug. 1998.

[5] M. A. Abou-Khousa, S. Kharkovsky, and R. Zoughi, "On the mutual coupling between circular resonant slots". in Proc. $3^{\text {nd }}$ Intern.Conf. on Electromagnetic Near-Field Characterization \& Imaging (ICONIC 2007), St. Louis, USA, 2007, pp. 117 - 122.

[6] D.M. Sheen, D.L. McMakin and T.E. Hall, "Three-dimensional millimeter-wave imaging for concealed weapon detection," IEEE Trans. Microw. Theory Tech., vol. 49, No. 9, Sept. 2001.

[7] T.J., Cui,, C.C.,Chew, A.A. Aydiner and C. Siyuan, "Inverse scattering of two-dimensional dielectric objects buried in a lossy earth using the distorted born iterative method" IEEE Trans. Geosci. Remote Sens., vol. 39, no. 2, pp. 339-346, Febr. 2001.

[8] S. Caorsi, A. Massa and M. Pastorino, "A crack identification microwave procedure based on a genetic algorithm for nondestructive testing" IEEE Trans. Antennas Propag, vol. 49, no. 12, pp. 1812 1820, Dec. 2001.

[9] S. Caorsi, G.L. Grangnani and M. Pastorino, "An electromagnetic imaging approach using a multi illumination technique" IEEE Trans. Biolog.l Eng., vol. 41, pp. 406-409, Apr. 1994. 


\title{
A Novel 24 GHz One-Shot, Rapid and Portable Microwave Imaging System
}

\author{
M.T. Ghasr ${ }^{1}$, M.A. Abou-Khousa ${ }^{1}$, S. Kharkovsky ${ }^{1}$, R. Zoughi ${ }^{1}$, and D. Pommerenke ${ }^{2}$ \\ 'Applied Microwave Nondestructive Testing Laboratory (amntl) \\ ${ }^{2}$ Electromagnetic Compatibility Laboratory \\ Electrical and Computer Engineering Department \\ University of Missouri-Rolla \\ Rolla, Missouri 65409
}

Tel: 1 (573) 341-4728, Fax: 1 (573) 341-6671

Email: m.t.ghasr@umr.edu

Keywords - electric field distribution, modulated scatterer technique, resonant slots, $2 D$ array, microwave imaging.

\section{INTRODUCTION}

Microwave imaging is based on measuring the relative scattered field from an object over a known two-dimensional (2D) space and then using various imaging algorithms (i.e., synthetic aperture, holographical, reconstruction techniques) to obtain geometrical and dielectric properties of the object (or its image) [1]. To this end, a scanned single probe or an array of probes sensitive to the electric field component of interest is used to measure the electric field over the 2D space (i.e., mapping the field). The basic probe design requires that its deployment does not significantly perturb the scatted field to be measured. Furthermore, the probe should be robust against parasitic reflections and coupling between probe array elements [2-3]. A probe which meets the above design requirements is one employing the modulated scatterer technique (MST). The use of MST for electric field measurements was first introduced in [3]. Since its introduction, MST with various linear scatterers has been extensively used for electric field measurement applications.

MST-based 2D array imaging systems have been designed and built with an array of subresonant dipoles at frequencies up to $12 \mathrm{GHz}$ (X-band) [2,4]. Although such designs use minimally perturbing elements and result in compact array for field sampling, they suffer from major drawbacks. The inefficiency of the sub-resonant dipoles, places their scattered signal very close to the noise floor of most detection systems. Furthermore, the mutual coupling among the dipoles can significantly limit system dynamic range. With increasing the frequency of operation, these problems render the implementation of such $2 \mathrm{D}$ imaging array very challenging.

In this paper, a novel MST-based 2D imaging system capable of rapid electric field mapping at higher operating frequencies is discussed. Modulated scatterer technique allows for spatially sampling (tagging) of the desired electric field. In other words, when the receiver demodulates an MST signal, it gives an output proportional to the magnitude and phase the incident electric field at the location of the modulated probe. Therefore, an array of modulated probes provides for electric field characteristics over a 2D space. Another feature of MST is that the effect of any other non-coherent signal such as clutter and noise on the measurement is significantly reduced [2]. The main feature of the proposed array design is high-Q compact resonant slots that are loaded with PIN diodes, and are then modulated either individually or simultaneously using 
orthogonal codes. To illustrate the efficacy of this design, a prototype array of 30 slots ( 6 by 5 ) with spacing of $\lambda_{0} / 2\left(\lambda_{0}\right.$ is the free space wavelength) and operating at a frequency of $24 \mathrm{GHz}$ was built and tested. A schematic of the prototype is shown in Figure 1.

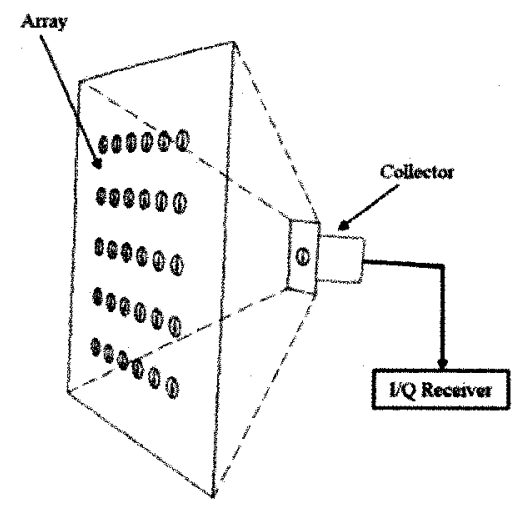

Figure 1: Schematic of the PIN diode-loaded array.

\section{IMAGING SYSTEM DESIGN AND MEASUREMENT APPROACH}

The design of the imaging system is based on the fact that switching speed and modulation depth are critical parameters when designing a rapid MST-based imaging system. To satisfy these requirements, high-Q resonant slots loaded with a fast switching PIN diode were used in the array design. The PIN diode switches in under $3 \mathrm{nS}$, while the slot goes from a very low insertion loss $(-20 \mathrm{~dB})$ to a very high insertion loss $(-2 \mathrm{~dB})$ slot when the diode switches [5], providing a large modulation depth with a relatively fast switching speed (i.e., rapid field measurement). The large modulation depth and the fact that coherent averaging over several modulated periods is readily possible, provides the system with a relatively high signal-to-noise ratio (SNR). In addition, the salient features of the slots are that they are efficient and exhibits very small mutual coupling [5]. Although, the longest dimension of the slot is less than $\lambda_{0} / 3$, it is resonated to work at the frequency of interest by special loading. Slot spacing of $\lambda_{0} / 2$ ensures adequate sampling of the field without sacrificing signal fidelity, which is adversely affected by mutual coupling [5]. The probe array (Figure 1) was manufactured using standard photolithographic PCB manufacturing technique on a Rogers 4350 board of 0.020 " $(0.5 \mathrm{~mm})$ thickness. The electric field scatted by the object to be imaged reaches the plane of the array and the respective electric field at the particular location of each slot is coupled into that slot. Subsequently, this signal is reradiated on the opposite side of the array. Using the PIN diodes, each slot is modulated at a certain modulation rate. In this way the electric field due to each slot can be individually discriminated and measured. This is the basis for using MST so that the scattered field from an object over a planar space can be unambiguously determined. To pickup the modulated scattered field from each slot, a collector consisting of a K-band rectangular waveguide with a resonant slot at its aperture is placed behind the array screen. This collector has the advantages of having a very small insertion loss, and a wide radiation pattern engulfing the array within its main lobe. Having a single collector allows the use of a single RF front-end without the use of complex microwave combiners. A combination of a relatively large planar array and a small collector, as shown in Figure 1, results in non-uniform coupling coefficients between each array element (slot) and the collector both in magnitude and phase. To correct for these coefficients, they are measured by applying a uniform plane-wave on the array. Since the measured field has known 
distribution (i.e., a uniform magnitude and phase distribution), any variation from that norm, is the sole effect of the array. Consequently, any subsequent measurement is referenced to the uniform plane wave measurement. Finally, an I/Q detector is used to measure the magnitude and phase of the captured re-radiated signal by each slot.

\section{MEASUREMENT AND DiscuSSIONS}

Several experiments were performed to asses the performance of the array probe. One of these experiments was set up as a through transmission configuration where the array probe is used as a receiver in the far-field region of a transmitting open-ended K-band rectangular waveguide. The distance between the transmitter and the array was set at $80 \mathrm{~mm}$. The electric field distribution due to the incident field radiated by the transmitting open-ended waveguide is measured and used as a reference to correct for the distortion caused by the array probe, as explained earlier. Then, a small metallic sphere ( $4 \mathrm{~mm}$ in diameter) was placed between the rectangular waveguide transmitter and the array probe receiver. Figure 2 shows the measured electric field distributions for when the sphere is $5 \mathrm{~cm}$ and $12 \mathrm{~cm}$ away from the slot array. Due to the limited number of sampling points (total of 30 pixels) of the array probe, the field distributions shown in Figure 2 was up-sampled 20 times and spatially interpolated to produce a more representative image of the electric field distribution. It must be noted that these images were measured and processed in real-time (under 15 milliseconds) which demonstrate the rapidity of this prototype imaging system. The indication of the sphere can be clearly seen in Figure 2a as a low intensity region (blue) showing the sphere casting its shadow on the array. This shadow indication is much larger than the sphere itself due to the distance from the sphere to the array. Furthermore, this indication is not circularly symmetric due to the rectangular geometry and limited number of sampling points on the array. Taking the sphere further away from the imaging array causes the sphere indication to appear larger as expected (Figure 2c-d). In addition, there is a high intensity spot in the center of the image shown in Figure $2 \mathrm{c}$ which is probably an indication of creeping waves since the small size of the sphere $(2 \pi \mathrm{r} / \lambda=1)$ places it in the Mie scattering region.

\section{SUMMARY}

A novel 2D microwave imaging system at $24 \mathrm{GHz}$ based on MST techniques utilizing high-Q resonant slots loaded with PIN diodes and a 30-element array probe prototype was designed and built. This imaging system is expected to produce images at a rate in excess of 30 images per second, be relatively small and portable. The results demonstrate the utility of the imaging system for high frequency electric field distribution mapping and high fidelity real-time imaging. Using this array probe not only the electric field distribution of scatterers, but also the near and/or far electric field distribution of antennas such as aperture antennas and monopoles may be imaged with high speed and in real time. This paper will present the detailed design of the entire imaging system along with results of measurements of electric field distribution due to various targets complex scattering and radiation from aperture antennas such as open-ended waveguides. Results of these measurements and a comparison between them and simulated electric field distribution will be presented and potential of this imaging system for other applications will be discussed in the proceedings paper. 


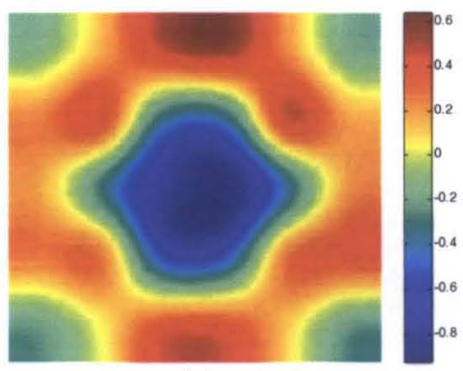

(a)

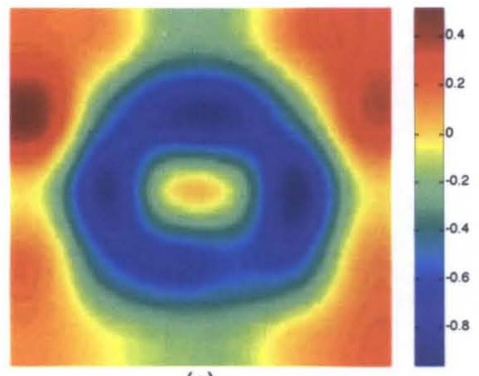

(c)

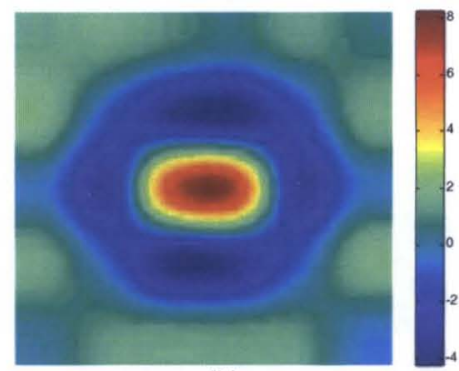

(b)

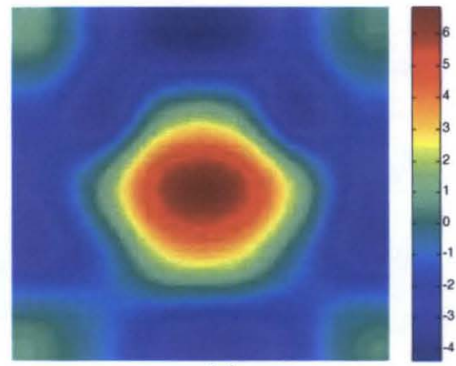

(d)

Figure 2: Magnitude (dB) and phase (deg.) of measured scattered electric field from a metallic sphere 4$\mathrm{mm}$ diameter metallic sphere at a distance of $5 \mathrm{~mm}$ from the array: a) magnitude, b) phase, and at a distance $12 \mathrm{~mm}$ from the array: c) magnitude, d) phase.

ACKNOWLEDGMENT: Funding for this work was provided by the NASA Marshall Space Flight Center, Huntsville, Alabama, USA.

\section{REFERENCES}

[1] Case, J.T., F. Hepburn and R. Zoughi, "Inspection of Spray on Foam Insulation (SOFI) Using Microwave and Millimeter Wave Synthetic Aperture Focusing and Holography," Proceedings of the IEEE Instrumentation and Measurement Technology Conference (IMTC06), pp. 2148-2153, Sorrento, Italy, April 24-27, 2006.

[2] J.-C. Bolomey, G.E. Gardiol, "Engineering applications of the modulated scatterer technique," Norwood, MA: Artech house INC. 2001.

[3] J. H Richmond, "A modulated scattering technique for measurement of field distributions," IEEE Transsactions on Microwave Theory and Techniques., vol. 3, no. 4, pp. 13-15, Jul 1955.

[4] A. Franchios, A. Joisel, C. Pichot, and J.-C. Bolomey, "Quantitative microwave imaging with a $2.45-\mathrm{GHz}$ planar microwave camera," IEEE Transactions on Medical Imaging, vol. 17, no. 4, pp.550-561, Aug 1998.

[5] M. A. Abou-Khousa, S. Kharkovsky, and R. Zoughi, "On the Mutual Coupling between Circular Resonant Slots", Proc. $3^{\text {nd }}$ International Conference on Electromagnetic Near-Field Characterization \& Imaging (ICONIC 2007), St. Louis, USA, 27 - 29 June 2007, pp. 117 122. 